\title{
Editorial
}

coloproctology $2020 \cdot 42: 373-374$

https://doi.org/10.1007/s00053-020-00485-2

(c) Springer Medizin Verlag GmbH, ein Teil von Springer Nature 2020

\section{Franc Hetzer}

Viszeralchirurgie Bellaria, Zürich, Schweiz

\section{Darmfunktionsstörung bei Querschnittlähmung}

In Deutschland leben ca. 140.000 Menschen mit Querschnittlähmung, und es treten jährlich rund 2300 neue Fälle auf, wobei zu $80 \%$ Männer betroffen sind. Die häufigste Ursache (ca. $70 \%$ ) sind Unfälle, überwiegend handelt es sich hierbei um Autounfälle. Nach einem Unfall wird der Patient im Idealfall in ein Querschnittzentrum gebracht, wo eine auf die Belange der Querschnittgelähmten abgestimmte medizinische Therapie darauf ausgerichtet ist, etwaige Folgeschäden frühzeitig zu erkennen bzw. zu vermeiden.

Man unterscheidet verschiedene Formen der Querschnittlähmung, abhängig von der Höhe der Rückenmarkverletzung. Von einer Paraplegie spricht man, wenn es zur Läsion des Rückenmarks im Brustwirbelbereich oder tiefer kommt. Eine Tetraplegie ist gekennzeichnet durch eine Schädigung im Bereich der Halswirbelsäule.

Seltener können Entzündungen das Rückenmark schädigen, allen voran die Poliomyelitis als infektiöse Erkrankung. Gegen diese Erkrankung gibt es zwar einen wirksamen Impfstoff, doch durch die zunehmende Impfmüdigkeit werden wieder vermehrt Fälle beobachtet. Auch die multiple Sklerose (MS) kann eine Entzündungsreaktion im Rückenmark auslösen, hier allerdings im Rahmen einer Autoimmunreaktion, d.h. einer Fehlregulation der Körperabwehr.

Auch eine Raumforderung durch einen Tumor oder einen Bandscheibenvorfall kann das Rückenmark verletzen. Eine Querschnittlähmung nach einer Operation der Halswirbelsäule ist selten, aber möglich, insbesondere im Rahmen von Komplikationen wie Nachblutungen und Wundinfektionen.
Darüber hinaus kann man zwischen einer kompletten und einer inkompletten Querschnittlähmung unterscheiden. Bei der erstgenannten Form kommt es zu einer vollständigen Durchtrennung der Nervenfasern im Rückenmark. Bei einer inkompletten Querschnittslähmung sind nicht alle Nervenfasern eines Rückenmarksegments geschädigt worden.

Der neurogenen Darmfunktionsstörung bei Querschnittlähmung liegt eine Schädigung des Rückenmarks oder der Cauda equina auf unterschiedlichem $\mathrm{Ni}$ veau zugrunde.

Veränderungen der gastrointestinalen Funktionen nach Querschnittlähmung sind in ihrer klinischen Erscheinung abhängig von der Höhe der Läsion, der Vollständigkeit der Verletzung und dem Zeitraum, der seit Eintritt der Querschnittlähmung vergangen ist. Von entscheidender klinischer Bedeutung sind die Veränderungen der Motilität des oberen und unteren Gastrointestinaltrakts sowie die Peristaltik, und auch die Frage, ob die Fähigkeit zur Entleerung des Rektums erhalten bleibt und der anale Verschlussmechanismus funktioniert.

Bezogen auf den zeitlichen Ablauf und den klinischen Verlaufkönnen die Veränderungen am Gastrointestinaltrakt nach einer Rückenmarkschädigung in 3 Phasen eingeteilt werden, wobei die Phasen fließend ineinander übergehen: Am Anfang spricht man von der Akutphase (spinaler Schock) mit Ausfall der Darmperistaltik (im Extremfall eine Paralyse). Nach Abklingen des spinalen Schocks setzt die Peristaltik wieder ein. Die weitere Entwicklung von intestinaler Motilität und Rektumfunktion ist nun von der Höhe und dem Ausmaß der Rückenmarkverletzung abhängig. Sie entscheidet über 
die Aktivität von Sympathikus und Parasympathikus sowie die Auslösbarkeit der Reflexsteuerung von Kolon und Rektum. Daraus können sich im klinischen Alltag im Langzeitverlauf schwerwiegende Komplikationen im Sinne von Megakolon, Paralyse und Ileus entwickeln.

\section{》) Ein gutes Darmmanagement beugt Komplikationen vor und hilft Betroffenen, ihren Alltag zu meistern}

Die involvierten Therapeuten müssen für jede Phase ein individuelles Darmmanagement-Programm erstellen. Es sollte allgemeine Maßnahmen wie ausreichende und angemessene Nahrungsund Flüssigkeitsaufnahme, Nahrungsergänzungsmittel und stuhlregulierende Medikamente beinhalten. Zusätzlich kommen Methoden zur Darmentleerung und Darmausräumung sowie physische Verfahren, stimulierende Mittel wie Zäpfchen, Einläufe oder Abführmittel zur Anwendung. Gegebenenfalls muss eine Stomaanlage zur Darmentleerung diskutiert werden. Ein gutes Darmmanagement beugt Komplikationen vor, und es hilft Betroffenen, ihren Alltag zu meistern.

Menschen mit einer Querschnittlähmung haben auch ein erhöhtes Risiko für Harnwegsinfekte. Sie sind ein maßgeblicher Grund für eine Hospitalisierung in wirtschaftlich gut gestellten Ländern und für einen vorzeitigen Tod in Entwicklungsländern. Ursächlich hierfür ist das Eindringen von Bakterien in die Blase, was durch Katheterisieren begünstigt wird. Andere Gründe können Restharn, Blasen- oder Nierensteine, eine überaktive Blase oder eine Abflussstörung des Urins (Reflux) sein.

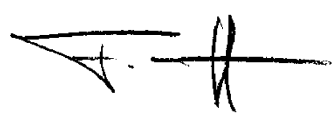

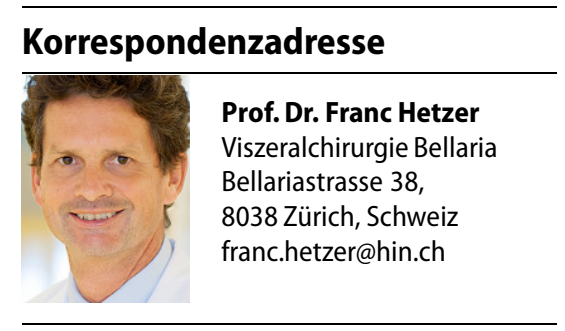

Interessenkonflikt. F. Hetzer gibt an, dass kein Interessenkonflikt besteht.

\section{Aktuelle Informationen}

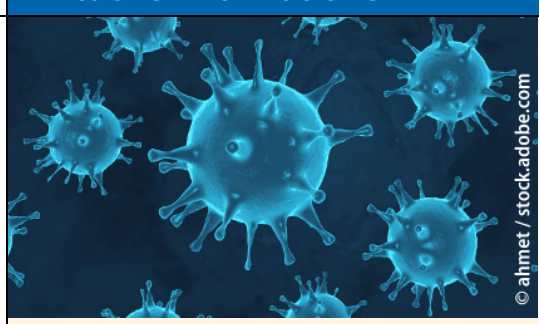

\section{Aktuelle Entwicklung zu COVID-19 bei Springer Nature und Springer Medizin}

Springer Nature und Springer Medizin unterstützen die globale Reaktion auf die COVID-19-Pandemie, indem ein schneller und direkter $\mathrm{Zu}$ gang zu den neuesten verfügbaren Forschungsergebnissen und Daten ermöglicht wird.

Auf der Homepage SpringerMedizin.de finden Sie ein immer aktuelles Dossier mit Beiträgen, Forschungsarbeiten und Ergebnissen zu SARS-CoV-2 sowie relevanten Links.

Darin z.B. auch die kürzlich publizierte

Empfehlung von DIVI, DGIIN, DGAI und DGP zur Intensivtherapie von Patienten mit COVID-19.

Springer Nature arbeitet mit globalen Organisationen zusammen, und verlinkt über SpringerNature.com/de auf eine eigene Landingpage mit einer Vielzahl an Information sowie freiem Zugriff auf die COVID-19-Contentplattformen von Nature Research, BioMed Central (BMC) und Springer.

Das Dossier zu Coronavirus / Covid-19 von Springer Medizin finden Sie hier: www.springermedizin.de/covid-19

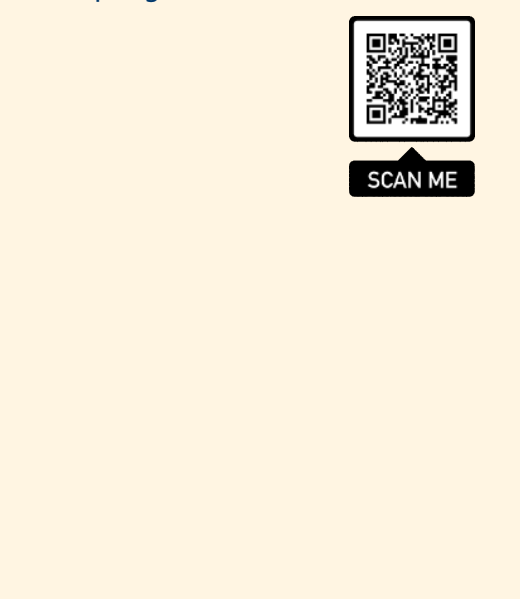

